\title{
A CASE REPORT OF BILATERAL BREAST REDUCTION MAMMOPLASTY CRISIS IN KUWAIT: MEDICAL MALPRACTICE OR POSTOPERATIVE COMPLICATION
}

\author{
Dina Galal Ibrahim*, Iman F. Gaballah, Mokhtar Fathy Abdel-Satar \\ Forensic Medicine and Clinical Toxicology Department, Cairo University, Egypt
}

*Corresponding author: Dina Galal Ibrahim

e-mail: dina.galal@kasralainy.edu.eg

Date of submission: 23 June 2020

Revised at: 22 August 2020

Accepted at: 28 August 2020

\begin{abstract}
Background: bilateral breast reduction mammoplasty is a popular cosmetic surgical operation. Patients usually aim to bring down the volume of the breast to achieve harmonious to their build along with overcoming the inconvenience caused by enormous, ill-shaped and suspended breasts. As the aesthetic appearance performs major importance in this procedure, a minimum of scar load is usually predicted, and the technique used must be individualized. However, preserving sensation and breastfeeding, especially during the child-bearing period, are also crucial. Complications are to be predicted in reduction mammoplasty; however, various post-operative complications were recorded because of errors of judgement, faulty surgical plan, and imprecise implementation of the plan. While plastic surgeons experience one of the highest percentages of physicians facing a malpractice claim, the unexpected cosmetic result presents the evident complaint. Objective and method: discussion of a case of a medical malpractice claim of post-operative bilateral necrosis resulted in total excision of the nippleareola complex (NAC) along with the underlying tissue in both breasts. Medical and operative history details of the patient were discussed against the surgical procedure and the surgeon's decision. The result is to understand the reason behind the catastrophic result and the management of the medicolegal claim to reach the prove of the medical litigation due to faulty surgical decision and management. Conclusion: most complications can be avoided with proper history taking, selection of the appropriate operation for the given patient and proper post-operative complications handling.
\end{abstract}

Keywords: Reduction mammoplasty, breast, malpractice, necrosis, complication.

\section{INTRODUCTION}

Breast reduction is one of the most successfully performed plastic operations. Many seek comfort during motion, reduction of neck, shoulders, and back pain, along with improvement of self-esteem (Stephen and Bradford, 1999).

The choice of the incision position and the pedicle to be used to preserve the nipple and areola are fundamental. Quadrants apart from the pedicle are removed, the breast reshaped, and redundant skin and tissue removed. Pre-operatively, detailed medical history regarding pregnancy, breastfeeding, disease, drug, alcohol, and smoking history should be provided in detail. Operative history, especially previous breast surgery, is foremost (Lakshmi and Jerry, 2013).

As regards physical examination, symptoms and signs of the huge pendulous breasts must be examined carefully. Detailed general and local (breast) physical examination usually provides necessary information to choose the right technique. Scar(s) of the previous breast operation(s) are fundamental clues for previous intervention(s). This must be provided with the surgical report(s) to demonstrate details of the previous operation, most importantly the pedicle providing the current blood supply for the nipple-areola complex 
(NAC) (Scott et al., 2005). Besides, a detailed, informed consent must be taken with all possible complications and results before the procedure.

Majority of litigation against plastic surgeons are mainly due to deficiency of informed consent, poor outcome, ugly scarring, or lack of experience. Breastsurgeries present almost a third of overall claims against plastic surgeons (Jena et al., 2011).

Multiple breast reduction techniques are practiced preserving the NAC. Inferior pedicle technique is a familiar technique used to maintain nipple sensitivity and preserve lactation after reduction (Rohrich et al., 2004). Ribeiro (2013) introduced a dermal-lipoglandular flap based on the inferior portion of the breast hump and used this tissue to "auto augment" the breast after excess tissue removal.

The superomedial pedicle was a modification introduced by Orlando and Guthrie (1975). Pedicle length is shortened and broadened to promote blood supply and innervation of the NAC. In this technique, the NAC is transferred on a superomedial de-epithelialized pedicle that has a thin subcutaneous tissue layer to preserve the dermal blood supply. The pedicle is based on the entire extent of the medial skin flap modelled after Wise incision and the whole novel nipple position apart from a small lateral section. The nipple is shifted laterally into place instead of upwards pedicle folding (Orlando and Guthrie, 1975).

Occasionally, a second reduction operation may be needed due to recurrent symptomatic macromastia due to hormonal therapy, weight gain, hormonal disturbance related to some diseases or as a drug side effect (Dancey, et al., 2008).

The uncertainty of the vascular pedicle used in the first reduction may complicate the choice of the second. Consequently, a modified central mound (MCM) reduction technique is usually used to maintain the remaining vascularity of the NAC in the central mound tissue along with superior and inferior vascular pedicle preservation (Joseph et al., 2019).

In this study, a faulty breast reduction mammoplasty operation that resulted in a total loss of the nipple-areola complex of both breasts is discussed, which is a catastrophic case of medical malpractice claim that was managed in the Forensic Medicine Department - Ministry of Interior - State of Kuwait.

\section{CASE PRESENTATION}

A malpractice claim has been raised by a 39year old female after 6 months of a bilateral breast reduction operation which resulted in the total loss of the nipple-areola complex (NAC) of both breasts. Her documented medical records were obtained. She had a history of hypothyroidism and asthma which are controlled by medication. eight years earlier, she had undergone the first breast reduction operation; however, details of the previous operation were not available because it was performed abroad.

After the first surgery, she commenced medications for induction of ovulation which resulted in an enlargement of her breasts. Consequently, she requested operative reduction mammoplasty due to symptomatic macrosomia. On her first presentation, the surgeon who was to operate the second reduction reported huge breasts with a longitudinal old scar in the midline of both breasts, from below the nipple to the lower breast fold. After a decision was taken to go for reduction surgery, informed detailed consent with possible complications and results was signed by the patient and a superomedial pedicle technique was planned.

On table, the superomedial pedicle was elevated and a Wise incision was done. The central pedicle was removed with the nipple and was shifted laterally followed by removal of $547 \mathrm{ml}$ of fat from the right breast and $551 \mathrm{ml}$ from the left. At the end of the operation, the capillary filling test was done which showed normal blood supply, and drains were put. 
Six hours after operation, congestion of both areolas was noticed; consequently, nitroglycerin spray was applied on the areolas. On the first postoperative day, capillary refilling test was normal although the congestion was worsened. On the 2nd postoperative day, an antibiotic was commenced with the continuation of nitroglycerine. On the 3rd postoperative day, necrosis started to appear on the skin and application of local creams and wound cleaning was done regularly. On the 5th postoperative day, nipple-areola complex (NAC) blackening was prominent, and necrosis reached $>50 \%$ of the NAC.

On the days that followed, necrosis progressed; antibiotic, silvadene and mebo creams were applied and a daily follow up was done; but superficial necrosis increased and, on the 3rd, postoperative week, the surgeon decided to proceed with the removal of the sloughed necrotic tissue since the NAC with the underlying tissue were no longer vital and on the $26^{\text {th }}$ postoperative day, the patient was taken to the operative theatre to remove the dead tissue. The NAC and part of the central pedicle of both breasts were totally removed, and after removal the breast was stitched longitudinally in the midline on both sides (fig. 1, 2) followed by a smooth postoperative healing.

Six months after the surgery, the patient raised a claim against the surgeon, and she was examined by the forensic team. Both breasts were similar, no masses were felt, and total removal of the nipple and areola was observed. A completely healed midline longitudinal scar, 15 and $13.5 \mathrm{~cm}$ in length were observed on the right and left breasts respectively. A completely healed transverse scar, 24 and $22 \mathrm{~cm}$ in length were seen on the underside of the right and left breasts, respectively.

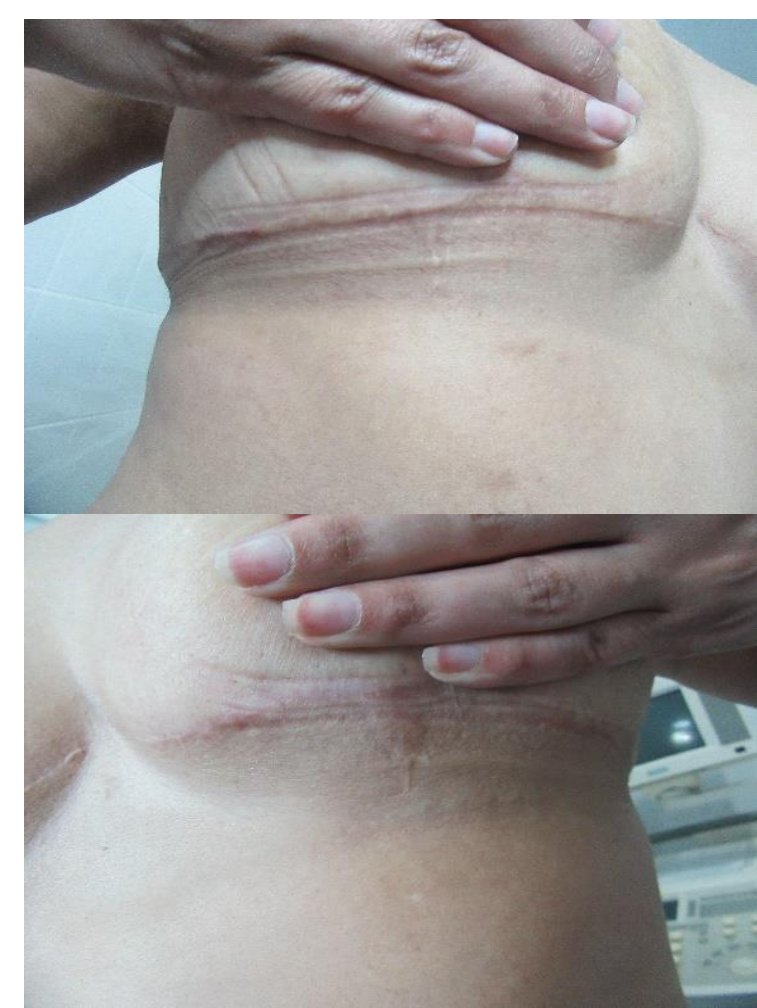

Figure (1): a transverse scar in the lower breast fold of the mammoplasty surgery on both sides.

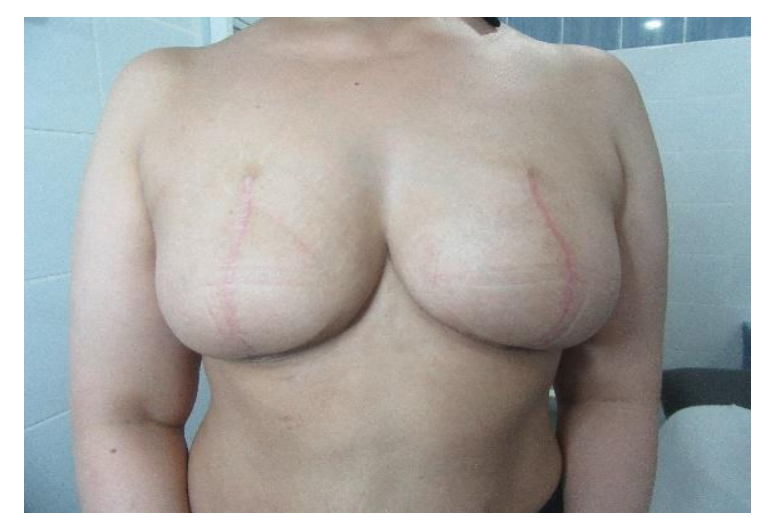

Figure (2): a longitudinal scar on the midline of both breasts.

The surgeon, who was a Serbian consultant expert in plastic surgery visiting Kuwait, admitted that the report of the first reduction operation had not been viewed before surgery. He mentioned that the inferior pedicle technique might have been used in the 1 st reduction. Consequently, the superomedial pedicle technique used in the second reduction led to the cutoff of the NAC blood supply. He reported that the patient could not access the reports of the previous reduction before operation and 
that NAC necrosis was one of the possible postoperative complications which were mentioned in the informed consent. The hospital and the operative theater where the operation took place were well equipped and reported as being fulfilling the basic needs for this kind of operation and pre and postoperative care.

Despite the necessity of the 2nd reduction operation, the case was reported as breach of medical assets, departure from the standard of care, and gross negligence followed by errors of commission and omission which caused direct damage to the patient. The malpractice allegation was that the defendant-plastic surgeon breached the applicable standard of care in performing cosmetic surgery through the selection of an inappropriate procedure. The chosen operation should have been based on the details of the previous operation which should have been obtained prior intervention to identify the blood supply of the NAC. Alternatively, the surgeon only utilized blood supply measurement and imaging (doppler sonar) before operation. Preservation of the blood supply and innervation of the NAC is crucial in breast reduction mammoplasty. Although the chosen reduction (superomedial pedicle technique) was an accepted breast reduction operation; it was not the appropriate choice for the patient.

Besides, the surgeon should have interfered as soon as the ischemic signs of the NAC started to appear through meticulous monitoring of the blood supply. As cyanosis persisted, the problem was identified and immediate intervention in the operating room could have salvaged the NAC. However, the result is not guaranteed in most cases. Compensation was approved by the court especially being in the childbearing period with a total loss of breast function on both sides apart from the significant disfigurement.

Also, acceptance of the patient for the NAC necrosis as a possible postoperative complication through an informed consent did not save the surgeon from a malpractice claim being raised due to a breach of the standard of care through omission of a meticulous preoperative preparation upon which the technique of the $2^{\text {nd }}$ reduction was to be based.

\section{DISCUSSION}

Despite being a proven method of treating macromastia, reduction mammoplasty is a challenging integration of aesthetic and functional plastic intervention. It must provide a secure and near expected result with, most importantly, nipple-areola complex (NAC) vitality preservation. The result must be judged on size, scar pattern, shape, symmetry of both sides, and nipple projection and position. As the perfect natural breast does not exist, the ideal operated breast must satisfy some aesthetic criteria (Nahai, 2010).

On the other hand, various complications are recorded after reduction mammoplasty. Hematoma, seroma, NAC necrosis and malposition, nipple retraction and loss of sensation, lactation problems, cancer, revision surgery, deformity, hypertrophied and symptomatic scars, fat necrosis, skin loss and delayed wound healing, nipple, and unsightly painful scars are the most experienced. Though, any breast intervention may result in such complications (Cunningham et al., 2005). The informed consent usually contains all these complications in detail, and that what the consent stated in our case. Consequently, the surgeon did not breach the right informed consent.

Post-reduction complications are almost always related to the patient, operative choice, and surgical experience. Regarding the patient, obesity, smoking, age, tissue resection weight per breast and radiation therapy are the most remarkable causes. However, judgement errors and wrong operative plans are the most significant causes related to the surgeon (Henry et al., 2009). 
Blood supply of the breast comes from perforators of two arterial systems, the superficial system which is composed of the lateral thoracic and internal mammary arteries and the deep system where the internal mammary artery (IMA) contributes considerably in the blood supply of the NAC (Hall-Findlay, 2011)

Two of the most preferred reduction techniques are the inferior and superomedial pedicle techniques. Both are named after the pedicle that contains the preserved vascular and nerve supply of the NAC which is removed as a flap to either the inferior or the superomedial direction respectively (Fig.3,4) (Wong et al., 2014).

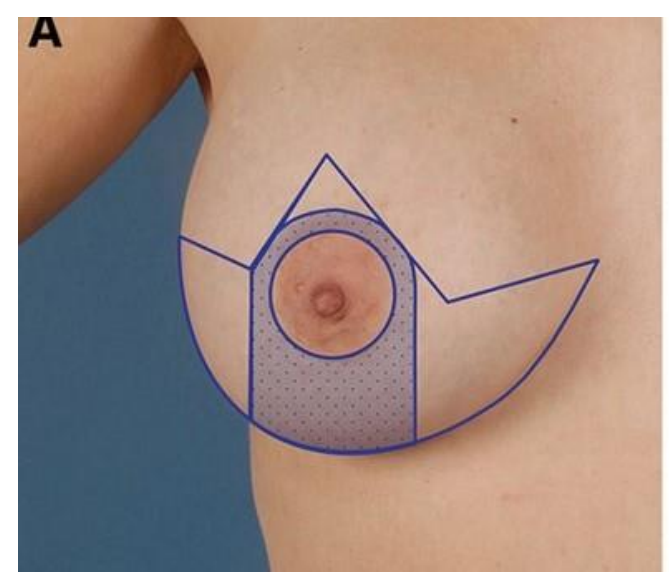

Figure (3): the inferior pedicle technique.

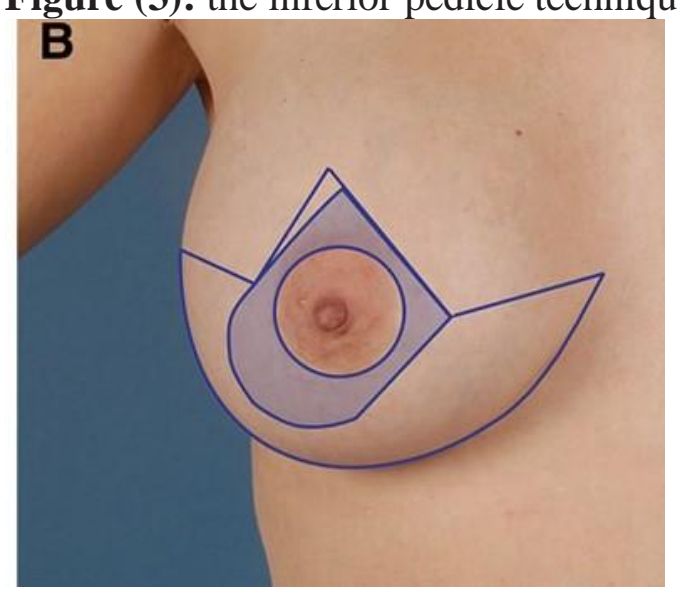

Figure (4): the superomedial pedicle technique.

In this case, the surgeon performed the superomedial pedicle technique without preoperative detection of the active blood supply of the NAC through a detailed medical report of the previous surgery or a vascular imaging technique such as Laser Doppler flowmetry, Doppler ultrasound and fluorescein angiography (Basaran et al., 2011). The surgeon believed that the disastrous result of the operation (total loss of the NAC and part of the central pedicle on both sides) indicated that the inferior pedicle was performed in the first operation. As a result, the blood vessels were severed in the second reduction operation.

Necrosis of the nipple is a dreaded complication, and the incidence varies and is related primarily to decreased vascularity of either the skin flaps or the pedicle in which the NAC is based. Based on that, early hours after surgery are critical. If color changes are noticed, immediate meticulous examination and intervention are then crucial to rescue the nipple. Sutures may be removed, and nipple vascularity reevaluated (Nahai, 2008). Nevertheless, the surgeon in this case did not perform any of the above which led to loss of the NAC vitality followed by total necrosis.

Cause in fact is fundamental to imposing liability in many kinds of medical malpractice actions (Meghaoury, et al. 2018). The term refers to the causal link between an act of commission or omission (by the surgeon) and the resulting damage or injury (to the patient) (Fischer, 1992). Compensation granted for medical malpractice must be given after approval of that the physician owed a professional duty to the patient and that the physician breached this duty by deviating from the standard of care, the patient was harmed through infliction of personal injury or wrongful death, and the harm inflicted was directly caused by the physician's breach of duty (Saleh and Masoud, 2019).

In this case, the surgery took place without enough knowledge of the anatomical blood supply of the breast; consequently, total necrosis of the NAC occurred that resulted in the loss of the breast function. The case presents a rare complication of bilateral NAC necrosis after a second reduction mammoplasty. The patient did not receive an optimal preoperative examination to ensure a good blood supply. Moreover, postoperative 
management of the complication was deficient which resulted in total loss of bilateral NAC and part of the central pedicle in both breasts for a 39-year-old female in the child-bearing period (Mavroforou, et al., 2004). Despite the preoperative agreement of the patient in the consent, the NAC necrosis in this case was believed to be a result of the inaccurate preoperative preparation and blind intervention.

\section{CONCLUSION}

This case presents an exceedingly rare postoperative result of bilateral NAC necrosis which is infrequently mentioned in literature. Awareness of breast anatomy, meticulous pre-operative designing, conservative tissue handling, and anticipatory post-operative attention will reduce the incidence of unfavorable results of the reduction mammoplasty.

\section{CONFLICT OF INTEREST}

There are not any financial, personal, or professional interests that could be construed to have influenced the work.

Funding Disclosure: Not applicable.

\section{REFERENCES}

Basaran, K.; Ucar, A.; Guven,E., Arinci,A.; Yazar,M. and Kuvat, S.V. (2011): Ultrasonographically determined pedicled breast reduction in severe gigantomastia. Plast. Reconstr. Surg.,128:252e-259e.

Cunningham, B.L.; Gear, A.J.; Kerrigan, C.L. and Collins, E.D. (2005): Analysis of breast reduction complications derived from the BRAVO study. Plast. Reconstr. Surg., 115:1597-604. Dancey, A.; Khan, M.; Dawson, J. and Peart, F. (2008): Gigantomastia - a classification and review of the literature. Journal of Plastic Reconstructive \& Aesthetic Surgery, 61(5):493-502

David A. Fischer (1992): Causation in Fact in Omission Cases. Utah. L. Rev., 1335.
Hall-Findlay, E.J. (2011): Aesthetic Breast Surgery: Concepts and Techniques. St. Louis: Quality Medical; Applied anatomy: key concepts for modern breast surgery. pp. 67-69.

Henry, S.L.; Crawford, J.L. and Puckett, C.L. (2009): Risk factors and complications in reduction mammaplasty: novel associations and preoperative assessment. Plast Reconstr Surg.,124:1040-1046.

Jena, A.B.; Seabury, S.; Lakdawalla, D. and Chandra, A. (2011): Malpractice risk according to physician specialty. N. Engl. J. Med., 365(7):629-636.

Joseph, R. S.; Patrick J.B. and Richard J.G.(2019:( Secondary reduction mammaplasty: does initial pedicle design matter?. Journal of Plastic Surgery and Hand Surgery, 53-105 :(2) .110

Lakshmi, S. and Jerry, R.j. (2013):Unfavourable results following reduction mammoplasty. Indian $\mathrm{J}$. Plast. Surg., 46(2): 401-407.

Mavroforou, A.; Giannoukas, A. and Michalodimitrakis, E. (2004): Medical litigation in cosmetic plastic surgery. Med Law, 23(3):479-488

Meghaoury, A.M.; Haggag, O.G.; Hindawi, R.F. and Farag, A.A. (2019): retrospective analytica

Nahai, F. (2010): The art of aesthetic surgery principles and technique, Quality Medical Publishing, 89(5): 558.

Nahai, F.R. (2008): MOC-PSSM CME article: Breast reduction. Plast. Reconstr. Surg.,121:1- 13.

Orlando, J.C. and Guthrie, R.H. (1975): The superomedial dermal pedicle for nipple transposition. Br. J. Plast. Surg., 28: $42-45$.

Ribeiro, L.A. (2013): A new technique for reduction mammoplasty. Plast. Reconstr. Surg., 55: 330-334.

Rohrich, R.J.; Gosman, A.A.; Brown, S.A.; Tonadapu, P. and Foster, B. (2004): Current preferences for breast reduction techniques: a survey of 
board-certified plastic surgeons. Plast. Reconstr. Surg., 114: 1724-1733.

Saleh, A.A. and Masoud, M. (2019): Comparative study of Knowledge and Practice Towards Medical Ethics Among physicians of Fayoum University Hospitals and General Fayoum Hospital, Egypt . Egypt J. Forensic Sci. Appli. Toxicol.,19(2):2941.

Scott, G.R.; Carson, C.L. and Borah, G.L. (2005): Maximizing outcomes in breast reduction surgery: A review of 518 consecutive patients. Plast. Reconstr. Surg., 116:1633-9.
Stephen, P.D. and Bradford, R. (1999):

Breast Reduction Techniques and Outcomes: A Meta-analysis. Aesthetic Surgery Journal, 19(4):293-303.

study of medical malpractice cases at Qalyubia Governorate 2010-2014. Egypt J. Forensic Sci. Appli. Toxicol.,18(1):79-92.

Wong, C.; Vucovich, M. and Rohrich, R. (2014): Mastopexy and Reduction Mammoplasty Pedicles and Skin Resection Patterns. Plastic and Reconstructive Surgery,2(8):e202. 


\section{دراسة حالة كارثيه لتصغير الثذيين: خطأ طبي أم مضاعفات ما بعد الجراحه}

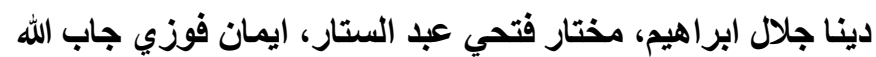

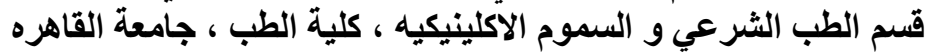

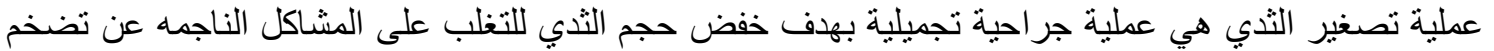

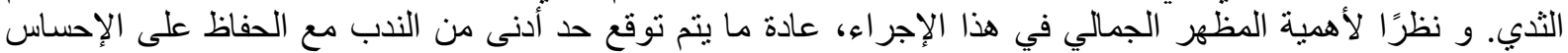

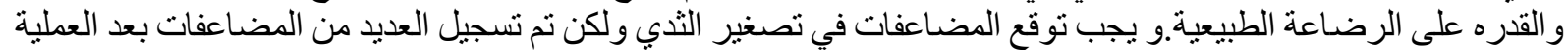

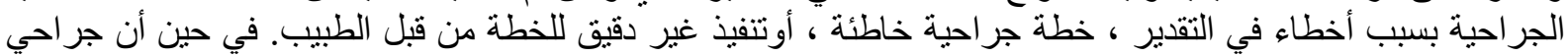

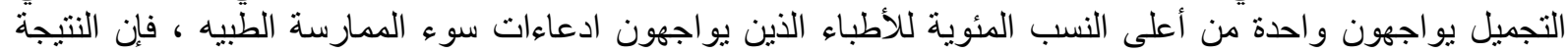

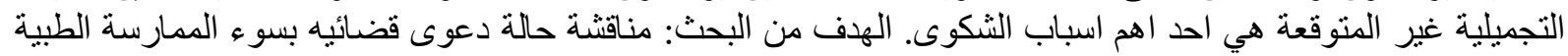

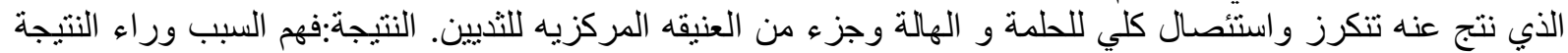

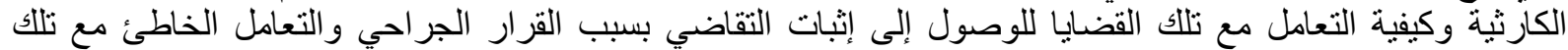

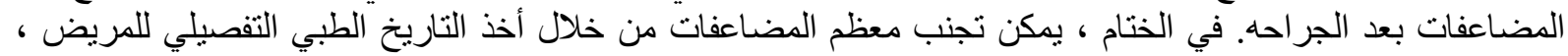

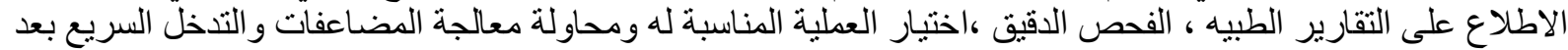

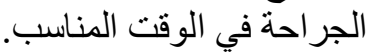

